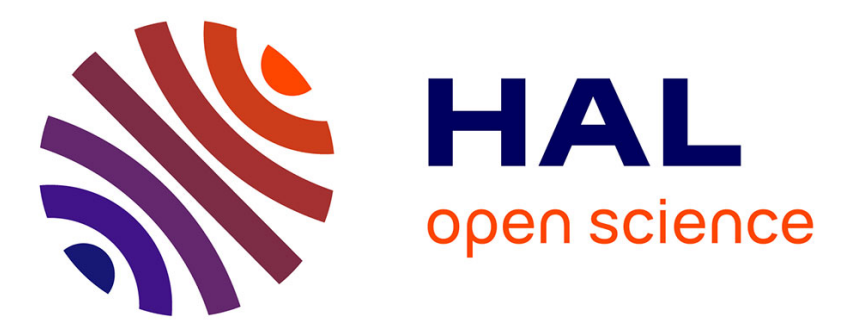

\title{
Possibilité d'une étape préliminaire à la mise à fleur chez une graminée. Effets de traitements par l'acide gibbérellique sur l'initiation florale et l'élongation de tiges de Bromus erectus Huds. sains et parasités par Ustilago hypodytes (Schlecht.) Fr
}

Evelyne Duval, Françoise Piton

\section{To cite this version:}

Evelyne Duval, Françoise Piton. Possibilité d'une étape préliminaire à la mise à fleur chez une graminée. Effets de traitements par l'acide gibbérellique sur l'initiation florale et l'élongation de tiges de Bromus erectus Huds. sains et parasités par Ustilago hypodytes (Schlecht.) Fr. Agronomie, 1985, 5 (10), pp.887-892. hal-00884717

\section{HAL Id: hal-00884717 https://hal.science/hal-00884717}

Submitted on 1 Jan 1985

HAL is a multi-disciplinary open access archive for the deposit and dissemination of scientific research documents, whether they are published or not. The documents may come from teaching and research institutions in France or abroad, or from public or private research centers.
L'archive ouverte pluridisciplinaire HAL, est destinée au dépôt et à la diffusion de documents scientifiques de niveau recherche, publiés ou non, émanant des établissements d'enseignement et de recherche français ou étrangers, des laboratoires publics ou privés. 


\section{Possibilité d'une étape préliminaire à la mise à fleur chez une graminée. Effets de traitements par l'acide gibbérellique sur l'initiation florale et l'élongation de tiges de Bromus erectus Huds. sains et parasités par Ustilago hypodytes (Schlecht.) Fr.}

Evelyne DUVAL \& Françoise PITON

Laboratoire des Membranes biologiques, Université Paris VII, Tour 54/53, $2^{e}$ étage, 2, place Jussieu, F 75005 Paris

L'effet de traitements par l'acide gibbérellique $\left(\mathrm{AG}_{3}\right)$ de Bromus erectus sains ou parasités par Ustilago hypodytes permet de mettre en évidence une étape préliminaire indispensable à la mise à fleur, jamais décrite jusqu'à présent.

Chez les plantes traitées par $\mathrm{AG}_{3}$ avant l'initiation florale cette étape préliminaire est inhibée en même temps que le stade double ride et ces plantes ne réagissent plus aux traitements ultérieurs par $\mathbf{A G}_{3}$. Par contre, les plantes parasitées qui ne forment pas de double ride sont encore capables de s'allonger et restent sensibles à la gibbérelline. Ces caractères intermédiaires entre l'état floral et l'état végétatif peuvent être expliqués si on suppose que le parasite permet le franchissement de l'étape préliminaire, inhibée par le traitement précoce par $\mathrm{AG}_{3}$. On doit alors admettre que le parasite n'agit pas par l'intermédiaire d'une gibbérelline.

Mots clés additionnels : Floraison, parasitisme, graminées, charbons. treatment of Bromus erectus Huds. either healthy or parasitized by Ustilago hypodytes (Schlecht.) Fr.

$\mathrm{AG}_{3}$ treatment of Bromus erectus, either healthy or parasitized by Ustilago hypodytes, suggested that there is an obligatory preliminary stage of flower initiation in this species, previously never described. When treated with $\mathrm{AG}_{3}$ before flower initiation, plants were unable to form double ridges or to reach this preliminary stage. They did not then react to later $\mathrm{AG}_{3}$ treatments. However, parasitized plants, which do not form double ridges, grew longer and remained sensitive to gibberellin. These intermediary characteristics between the flowering and vegetative stages can be explained by supposing that the parasite allows the plant to bypass the above-mentioned preliminary stage, which is inhibited by early treatments by $\mathrm{AG}_{3}$. This implies that the parasite does not act via gibberellic acid.

Additional key words : Flowering, parasitism, grasses, smut.

\section{INTRODUCTION}

Chez une graminée fourragère, Bromus erectus Huds., la présence d'un champignon parasite, Ustilago hypodytes (Schlecht.) Fr., inhibe l'initiation florale. Les tiges qui normalement auraient dû fleurir ne portent que des feuilles (Duval et al., 1981). Elles ne sont cependant pas maintenues dans un état véritable- ment végétatif, car elles subissent une forte élongation (Piton et al., 1982). A la surface de ces tiges se forment les spores charbonneuses.

On peut formuler l'hypothèse que, chez les plantes parasitées, une étape préliminaire de la mise à fleur est franchie mais ne peut être dépassée. L'étude des modalités d'action de ce parasite peut donc apporter des informations sur les mécanismes de la floraison. 
Dans ce but, nous avons cherché soit à reproduire les effets du parasite sur des plantes saines, soit à modifier ses effets chez des plantes parasitées, par divers traitements connus pour leur activité sur la croissance et la mise à fleur.

Nous rapportons ici les résultats de traitements par l'acide gibbérellique, $\mathrm{AG}_{3}$.

\section{MATÉRIEL ET MÉTHODES}

\section{A. Matériel biologique}

Des expériences préliminaires, réalisées sur des plantes issues de graines de provenances diverses, ont montré l'efficacité des traitements par pulvérisation de solutions aqueuses d' $\mathrm{AG}_{3}$ et ont permis de définir les concentrations convenables.

Les expériences rapportées dans cet article ont été réalisées sur un matériel génétiquement homogène constitué par un clone de plantes obtenu par séparation des talles et comportant un lot sain et un lot parasité par $U$. hypodytes (DUVAL et al., 1981).

La séparation des talles s'effectue en septembre. Celles qui sont de vigueur et de rang équivalents sont sélectionnées et repiquées à raison de une par pot dans un mélange volume à volume de sable de rivière et de compost de feuilles. Pendant la durée de l'expérience, ces tiges sont maintenues en culture monotalle par suppression régulière des bourgeons axillaires ce qui implique l'élimination des feuilles les plus âgées.

\section{B. Conditions de culture et traitements}

Les plantes sont cultivées en serre non chauffée, à la lumière du jour.

Les traitements par $\mathrm{AG}_{3}$ sont effectuées par pulvérisation de $1 \mathrm{ml}$ de solution aqueuse sur chaque plante une fois par semaine. Deux concentrations sont utilisées : $10^{-4}$ et $5 \times 10^{-4} \mathrm{~g} \cdot \mathrm{ml}^{-1}$. Une $1^{\text {re }}$ série de traitements ( $\mathrm{T} 1)$ est commencée en décembre alors que les plantes sont à l'état végétatif. Une $2^{\mathrm{e}}$ série de traitements (T2) est réalisée à partir de l'initiation florale, en mars, sur des plantes jusqu'alors non traitées. Les 2 traitements se poursuivent jusqu'à l'anthèse. Pour chaque traitement, dans chaque série, on dispose initialement d'au moins 15 plantes.

L'évolution des apex est étudiée régulièrement par prélèvement et dissection de 5 plantes dans une population témoin ou en fin de traitement.

Pour situer l'initiation, nous avons choisi d'utiliser la date pour laquelle 3 au moins des 5 plantes disséquées présentaient le stade double ride. Ce stade correspond au stade B dans l'échelle de GAY \& MENÉTRIER (1978) et au stade Ae de JONARD et al. (1952).

Pour situer l'épiaison que nous définissons par l'apparition du sommet de l'inflorescence au-dessus de la gaine de la dernière feuille (BLONDON, 1968), nous avons choisi la date pour laquelle 50 p. 100 des individus ont atteint le stade recherché ; ce critère, qui a le défaut de correspondre au moment où la variabilité de pourcentage de réalisation est maximale, a l'avantage d'être couramment utilisé (DELECOLLE \& GURNADE, 1980).
De même pour l'anthèse, le stade est considéré comme atteint lorsqu'au moins 50 p. 100 des plantes présentent des étamines sorties.

\section{RÉSULTATS ET DISCUSSION}

\section{A. Effet des traitements sur l'initiation florale}

En serre non chauffée, l'initiation florale a lieu dans le courant du mois de mars. Dans ces conditions, le traitement précoce par $\mathrm{AG}_{3}$ (T1) à la concentration de $5 \times 10^{-4} \mathrm{~g} \cdot \mathrm{ml}^{-1}$ provoque une inhibition totale de l'initiation florale. Cette inhibition se retrouve également pour 13 plantes traitées par $\mathrm{AG}_{3}$ à la concentration $10^{-4} \mathrm{~g} \cdot \mathrm{ml}^{-1}$. Cependant 2 des plantes de ce lot ont différencié des ébauches florales, mais avec un retard de 2 mois sur les plantes témoins.

Du point de vue de l'initiation florale, les effets des traitements par $\mathrm{AG}_{3}$ sont donc semblables à ceux du parasitisme (Planche 1).

\section{B. Effets des traitements sur l'élongation des tiges}

Chez le brome dressé, l'élongation commence par une croissance acropète ( $1^{\text {re }}$ étape) qui se situe après' l'initiation ; l'allongement des tiges commence par les entre-nœuds les plus proches du rhizome. Les tiges comportent alors des entre-nœuds d'autant plus petits qu'ils sont plus proches de l'inflorescence. Ce type de croissance a été récemment modélisé chez le blé (MALVOISIN, 1984). Il se poursuit régulièrement jusqu'à l'épiaison.

A l'épiaison, il reste sous le rachis, déjà bien développé, des entre-nœuds très petits qui sont encore dans l'état défini par MALVOISIN comme état de croissance modérée.

A partir de ce stade, un changement brusque se produit en ce qui concerne l'élongation ( $2^{\mathrm{e}}$ étape). Les entre-nœuds subissent un allongement beaucoup plus rapide et d'autant plus prononcé qu'ils sont plus proches de l'inflorescence, phénomène qui a été décrit chez de nombreuses graminées (PRAT, 1948).

\section{Effets des traitements sur l'élongation des plantes saines jusqu'à l'initiation}

Le traitement T1 stimule l'élongation des tiges pour la concentration la plus élevée (fig. 1).

Pendant cette période, dans les conditions où nous avons travaillé, la tige saine se montre donc sensible à $\mathrm{AG}_{3}$. La croissance très réduite des témoins s'expliquerait alors par une synthèse insuffisante $d^{\prime} A^{\prime} G_{3}$.

Entre la période d'initiation (fin mars) et la période d'épiaison, ( $1^{\text {re }}$ étape), les effets d'un traitement par la gibbérelline sont très différents suivant qu'ils ont été appliqués à des plantes initiées ou non (fig. 2).

Les plantes dont l'initiation a été bloquée par le traitement précoce par $\mathrm{AG}_{3}$ (T1) voient leur élongation s'arrêter. Il ne s'agit pas d'une entrée en dormance de l'apex : l'apparition des feuilles, bien que plus lente, se poursuit chez les plantes traitées (fig. 3 ). Les plantes dont la croissance est la plus ralentie étant celles qui reçoivent la plus faible dose d' $\mathrm{AG}_{3}$, on ne saurait attribuer cet arrêt de croissance à un effet inhibiteur direct de la gibbérelline. 


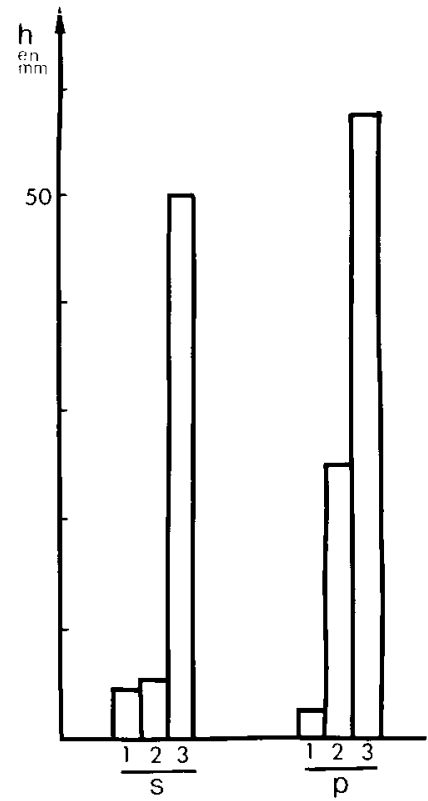

Figure 1

Longueur moyenne des tiges ( $h$, en $\mathrm{mm}$ ) au moment de l'initiation chez les plantes saines $(S)$ et parasitées $(P)$ : témoins $(1)$, traitées par $A G_{3} 10^{-4} \mathrm{~g} . \mathrm{ml}^{-1}(2)$, traitées par $A G_{3} 5.10^{-4} \mathrm{~g} . \mathrm{ml}^{-1}$ (3).

Stem length at initiation ( $h$, mean in $\mathrm{mm}$ ) in healthy (S) and parasitized $(P)$ plants : untreated (1), treated with $A G_{3}$ at $10^{-4} \mathrm{~g} . \mathrm{ml}^{-1}$ (2), treated with $\mathrm{AG}_{3}$ at $5.10^{-4} \mathrm{~g} . \mathrm{mI}^{-1}$ (3).

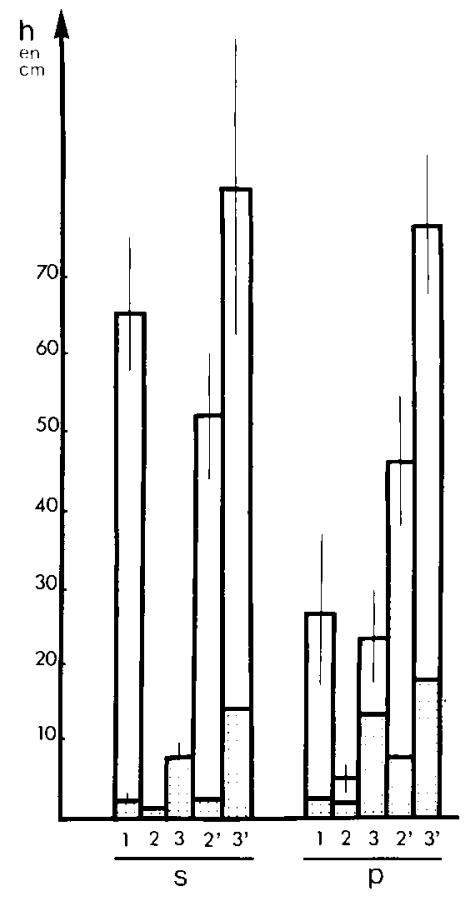

Figure 2

Longueur moyenne des tiges ( $h$, en $\mathrm{cm}$ ) lors de l'épiaison au moment de l'anthèse $\square$ chez les plantes saines (S) et parasitées $(P)$ : témoins $(1)$, traitées avant l'initiation par $A G_{3} 10^{-4} \mathrm{~g} . \mathrm{ml}^{-1}$ (2) ou par $A G_{3} 5.10^{-4} \mathrm{~g} \cdot \mathrm{ml}^{-1}$ (3), traitées après l'initiation par $A G_{3}$ $10^{-4} \mathrm{~g} \cdot \mathrm{ml}^{-1}\left(2^{\prime}\right)$ ou par $A G_{3} 5.10^{-4} \mathrm{~g} \cdot \mathrm{ml}^{-1}\left(3^{\prime}\right)$.

$L$ 'intervalle de confiance est représenté par un trait vertical.

Stem length at earing $\square$ and anthesis $\square(h$, mean in $(\mathrm{cm})$ in healthy (S) and parasitized $(P)$ plants: untreated (1), treated with $A G_{3}$ at $10^{-4} \mathrm{~g} \cdot \mathrm{ml}^{-1}$ (2) or with $A G_{3}$ at $5 \cdot 10^{-4} \mathrm{~g} \cdot \mathrm{ml}^{-1}$ (3) before initiation, treated with $A G_{3}$ at $10^{-4} \mathrm{~g} \cdot \mathrm{ml}^{-1}\left(2^{\prime}\right)$ or with $A G_{3}$ at $5.10^{-4} \mathrm{~g} \cdot \mathrm{ml}^{-1}\left(3^{\prime}\right)$ after initiation.

The vertical lines represent the confidence intervals of the means.

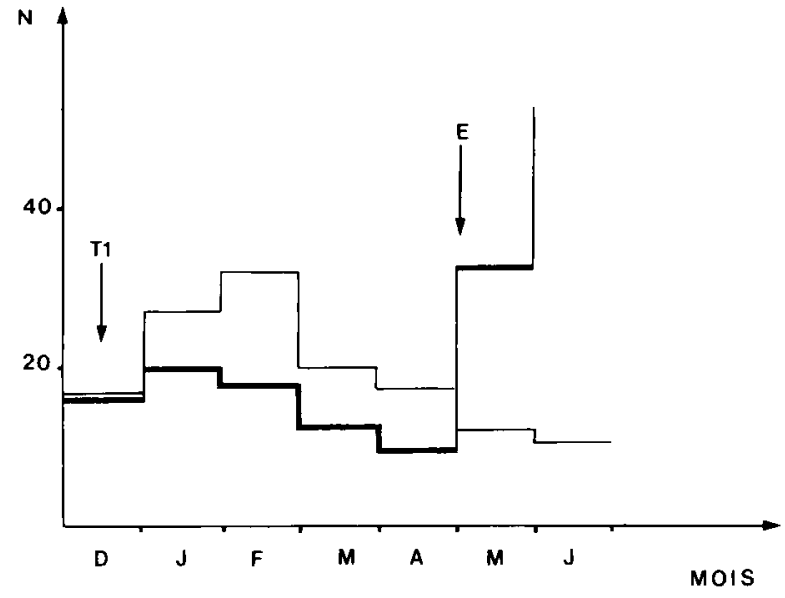

Figure 3

$L a$ variation du nombre de jours ( $N$ ) nécessaire à l'émergence d'une feuille hors des gaines foliaires en fonction du temps en mois est représentée par un trait épais (__ ) pour les plantes saines témoins et par un trait mince (-) pour les plantes traitées par $A G_{3} 10^{-4} \mathrm{~g} . \mathrm{ml}^{-1}$ avant l'initiation.

$T 1$ indique le début du traitement qui inhibe l'initiation mais on note qu'il ne provoque pas la dormance de l'apex : la formation de jeunes feuilles est alors ralentie mais non arrêtée. E indique la période de l'épiaison (émergence de la panicule hors des gaines foliaires pour au moins 50 p. 100 des plantes).

Variation with time (in months) in the number of days $(N)$ necessary for the emergence of a young leaf in healthy control plants $\left(\frac{10^{-4} \mathrm{~g} . \mathrm{ml}^{-1}}{)}\right.$, and treated before floral initiation with $\mathrm{AG}_{3}$ at

$T I$ indicates the beginning of treatment which inhibits initiation but does not provoke dormancy of apices : the formation of young leaves is then slowed down but not stopped. E indicates the earing time.

Chez les plantes dont l'apex est initié préalablement au traitement (T2), la croissance est stimulée par des applications $\mathrm{d}^{\prime} \mathrm{AG}_{3}$ à $5 \times 10^{-4} \mathrm{~g} \cdot \mathrm{ml}^{-1}$.

On peut se demander pourquoi la gibbérelline devient inactive sur l'élongation des tiges dont l'initiation a été bloquée alors qu'elle agissait avant la période d'initiation et qu'à forte dose elle continue d'agir sur les plantes initiées.

Une des hypothèses possibles est que la tige exige alors davantage de gibbérelline pour s'allonger, ce qui revient à dire qu'à cette période les tiges sont moins sensibles à $\mathrm{AG}_{3}$. Il faut admettre que, dans les plantes initiées, le supplément de gibbérelline nécessaire est fourni par l'apex floral. Dans cette hypothèse, les traitements par $\mathrm{AG}_{3}$ des plantes initiées devraient provoquer une stimulation de l'élongation des tiges en ajoutant leurs effets à ceux des gibbérellines endogènes, ce qui se vérifie pour la concentration la plus élevée. Une autre hypothèse serait que la sensibilité à la gibbérelline reste la même mais que d'autres facteurs, nécessaires pendant cette période, viennent stimuler la croissance des tiges dont l'apex est initié et ajoutent leurs effets à ceux des traitements. L'absence de ces facteurs chez les apex non initiés expliquerait la croissance limitée.

Après l'épiaison ( $2^{e}$ étape), la partie supérieure de la tige devient pratiquement insensible aux traitements par $A G_{3}$. Deux explications sont possibles : soit que cette phase d'élongation ne soit pas dépendante de la gibbérelline, soit que la plante produise elle-même des quantités de gibbérelline telles que les doses ajoutées par les traitements sont négligeables. 

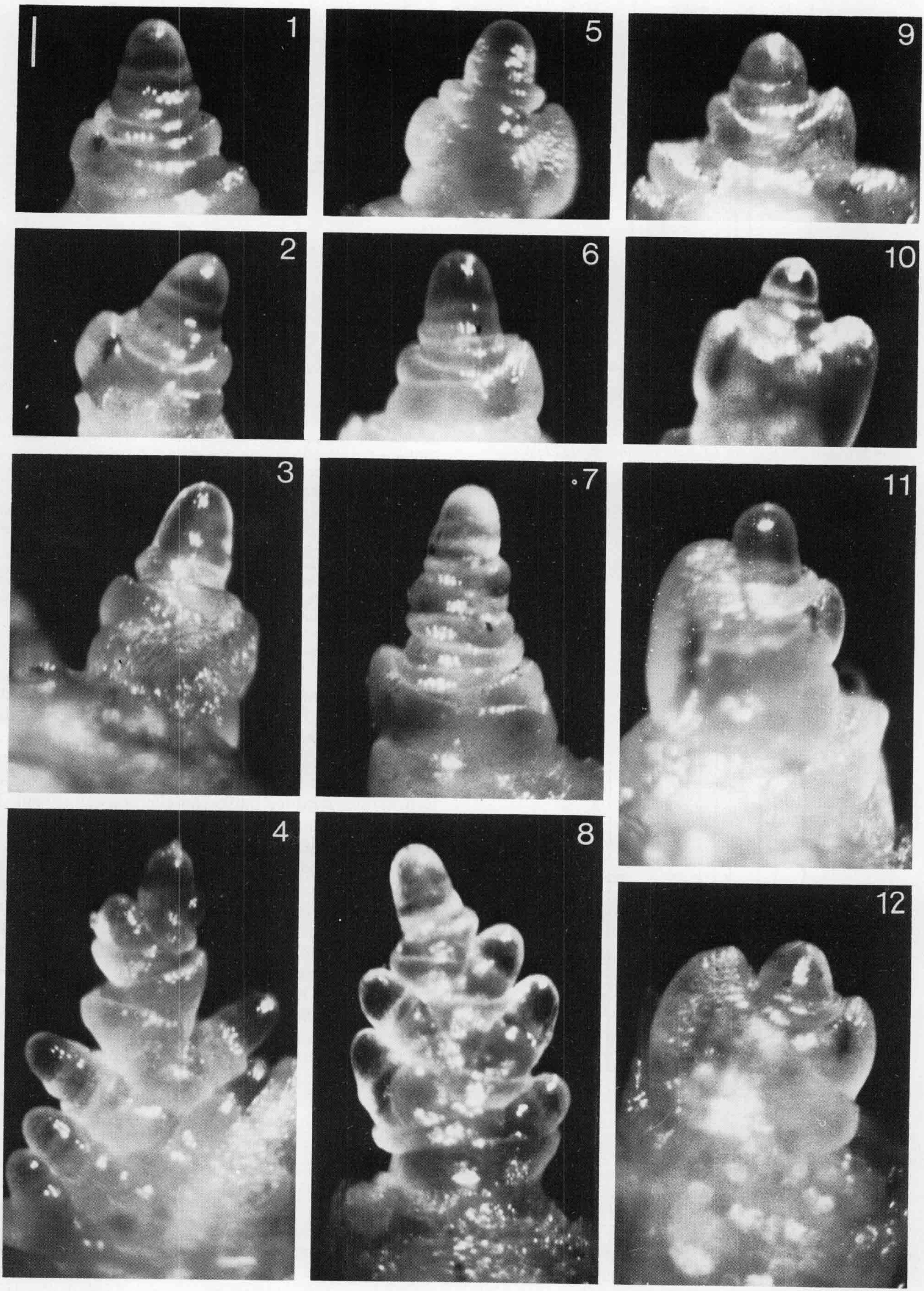
Planche 1

Effets de traitements par 2 concentrations différentes d'AG ${ }_{3}$, commencés à différents moments de l'évolution de bromes dressés sains et parasités (la barre d'échelle représente $0,1 \mathrm{~mm}$ ).

Apex des plantes saines après traitement précoce (TI) par $A G_{3}$ $5 \times 10^{-4} \mathrm{~g} \cdot \mathrm{ml}^{-1}$ : en février (1), en mars (2), en juin (3). Après un traitement précoce par $\mathrm{AG}_{3} 10^{-4} \mathrm{~g} . \mathrm{ml}^{-1}$, différenciation florale tardive et anormale observée dans quelques cas (4).

Apex des plantes saines témoins : en février (5), début mars (6); début avril, stade double ride observé pour $50 \mathrm{p} .100$ au moins des plantes (7) ; fin avril, stade primordia observé chez tous les témoins (8).

Apex des plantes parasitées témoins : en février (9), en juin (10). Apex des plantes parasitées après un traitement précoce par $10^{-4}$ g. $\mathrm{ml}^{-1}$ en avril (11), en juin (12).

Effects, at different times in the development of Bromus erectus either healthy or parasitized, of two different concentrations of $A G_{3}$.

Apices of healthy plants after early treatment (Tl) by $A G_{3}$ $5 \times 10^{-4} \mathrm{~g}_{\mathrm{ml}}^{-1}$ in February (1), March (2) and June (3). After an early treatment with $\mathrm{AG}_{3}$ at $10^{-4} \mathrm{~g} . \mathrm{ml}^{-1}$, late and irregular floral differentiation was observed in some cases (4).

Apices of control healthy plants in February (5), and early March (6) ; early in April the double-ridge stage was observed in $50 \%$ of the plants at least (7); at the end of April, primordia stage was observed in all control plants (8).

Apices of parasitized control plants in February (9) and June (10). Apices of parasitized plants after early treatment with $A G_{3}$ at $10^{-4} \mathrm{~g} . ~^{\mathrm{ml}^{-1}}$ in April (Il) and June (12).

\section{Effets des traitements sur l'élongation des plantes parasitées}

Pendant la période qui précède l'initiation (de décembre à fin mars), les témoins présentent une élongation réduite à quelques $\mathrm{mm}$. Le traitement par $\mathrm{AG}_{3}$ stimule l'élongation des tiges d'autant plus que la concentration utilisée est plus forte (fig. 1).

A ce stade, la tige est donc sensible à la gibbérelline comme celle des plantes saines, mais l'effet des traitements est plus fort. Cette différence pourrait s'expliquer en admettant que la présence du parasite provoque l'apparition de facteurs de croissance différents des gibbérellines. Dans ces conditions, l'apport d' $\mathrm{AG}_{3}$, interférant avec ces facteurs endogènes, permettrait une élongation supérieure des tiges chez les témoins; le taux de ces facteurs endogènes serait suffisant pour inhiber l'initiation mais trop faible pour provoquer l'élongation des entre-nœuds.

Après la période d'initiation, on constate une nette différence de réaction entre les 2 séries de traitements (fig. 2).

Dans la série $\mathrm{T} 1$, jusqu'au moment de l'épiaison ( I $^{\text {re étape) }}, \mathrm{AG}_{3}$ à forte dose stimule l'élongation de la tige. Après l'épiaison ( $2^{\mathrm{e}}$ étape), quelle que soit la concentration d' $\mathrm{AG}_{3}$ utilisée et contrairement à ce qu'on observe chez les graminées saines traitées, la croissance se poursuit mais devient inférieure au témoin. Au total, en fin de traitement, la longueur des tiges traitées est inférieure ou égale à celle des témoins. Nous avons parallèlement observé un retard voire une inhibition totale de la sporulation.

On constate donc une réaction analogue à ce qui s'observe chez les plantes saines. Là encore un effet inhibiteur direct de la gibbérelline ne peut être retenu, la concentration la plus forte étant la moins défavora- ble à la croissance. Comme dans le cas des plantes saines, on doit supposer qu'il y a, après la période d'initiation (laquelle n'a pourtant pas eu lieu), un changement des besoins de la plante en facteurs de croissance.

Cependant, chez les plantes parasitées, l'inhibition de la croissance est moins forte que chez les plantes saines. On doit forcément attribuer cette différence de réaction à la présence du parasite ce qui corrobore l'hypothèse d'une augmentation de la teneur en substances actives sur la croissance due au champignon.

Dans la série T2, en fin de traitement, les tiges sont plus hautes que les témoins. Dans ce cas la gibbérelline est donc nettement stimulatrice de l'élongation des entre-nœuds.

Cette différence de comportement, dans les mêmes conditions externes, entre la série $T 1$ et la série $T 2$ ne peut trouver d'explication que dans un changement d'état de tout ou partie de la plante parasitée au moment de l'initiation. Ce changement d'état, nécessaire à une élongation normale de la tige et à la sporulation, intervient avant ou pendant la période d'initiation et est inhibé par le traitement précoce à la gibbérelline ; il ne se traduit par aucune modification morphologique de l'apex.

On voit alors qu'il n'est pas possible d'admettre que le parasite, qui permet ce changement d'état, agisse par l'intermédiaire d'une gibbérelline, comme les différences d'élongation entre les plantes saines et parasitées traitées avant l'initiation l'avaient laissé supposer. D'autres médiateurs doivent être recherchés.

\section{CONCLUSIONS}

Le parasite empêche l'initiation florale mais permet une certaine élongation des tiges. Par contre, si l'initiation florale est inhibée par $\mathrm{AG}_{3}$, il n'y a pas d'élongation.

Chez les plantes saines restées à l'état végétatif sous l'influence de l' $\mathrm{AG}_{3}$, la gibbérelline ne peut reproduire le type de croissance observée chez les plantes parasitées. Celles-ci présentent donc des potentialités que n'ont pas les plantes saines traitées précocément par $\mathrm{AG}_{3}$. L'analogie de comportement entre les plantes saines et parasitées de la série Tl conduit à admettre que la gibbérelline appliquée avant l'initiation florale empêcherait le franchissement d'une étape préliminaire.

Cette étape $\mathrm{P}$ serait nécessaire à l'initiation comme à l'élongation des tiges saines et parasitées et à la sporulation. Elle ne se traduirait par aucun changement morphologique de l'apex (fig. 4).

Puisque le parasite ne peut agir à la manière d'une gibbérelline, nous formulerons l'hypothèse qu'il provoque l'apparition d'un facteur inhibant l'initiation florale proprement dite sans perturber l'étape préliminaire $\mathrm{P}$. Ce facteur pourrait ajouter ses effets à ceux des traitements expérimentaux pour stimuler la croissance des tiges. Il serait intéressant de savoir si ce facteur, différent des gibbérellines, joue également un rôle dans la croissance ultérieure des tiges. 

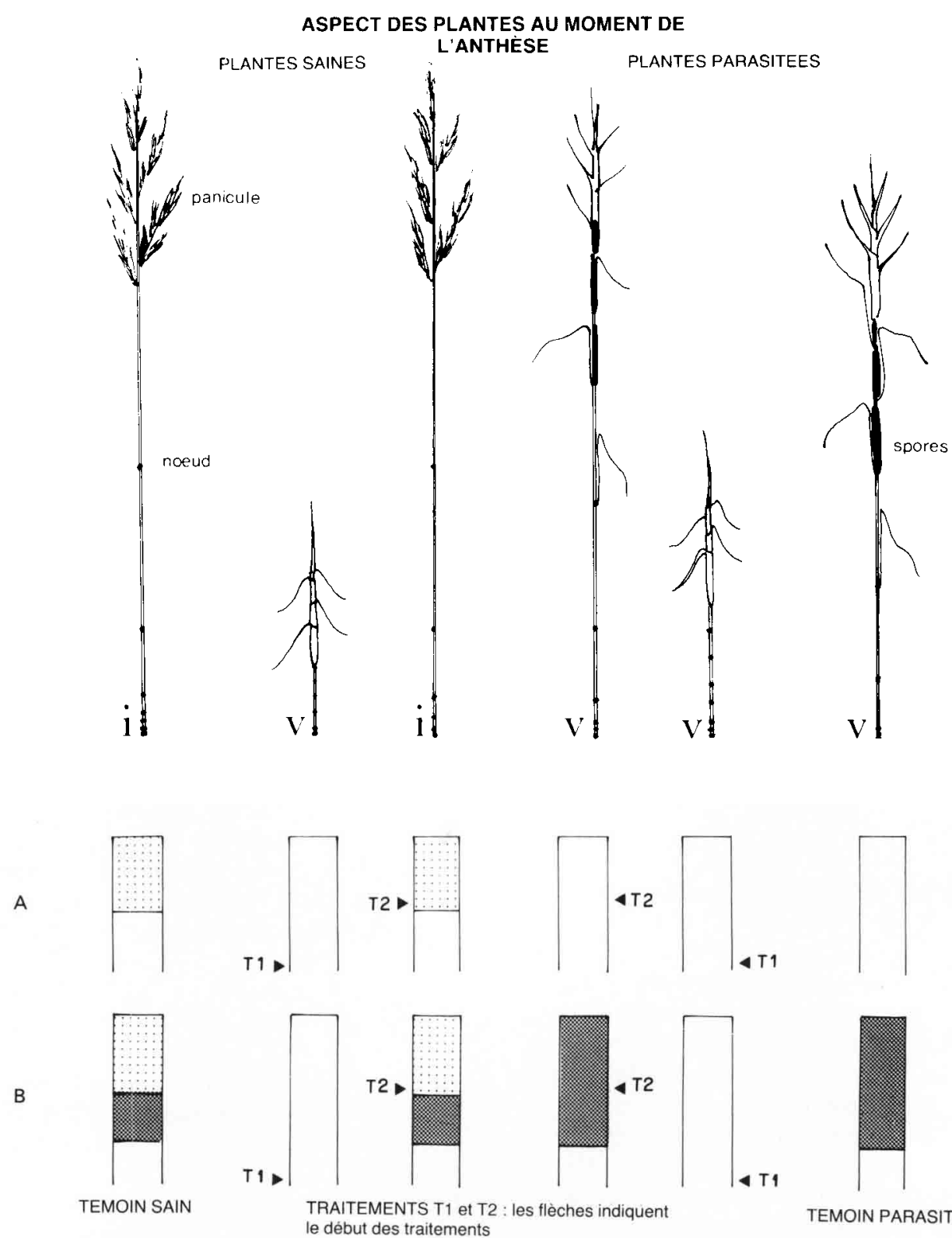

TEMOIN PARASITE

Figure 4

Représentation schématique des résultats expérimentaux et de leur interprétation :

Diagram representing experimental results and their interpretation : $V$ : apex végétatif; vegetative apex.

$I$ : apex initié (stade double ride) ; initiated apex (double ridge stage). Initiation.

Etape préliminaire; preliminary stage.

Etat végétatif; vegetative condition.

\section{Interprétation}

Selon les données actuellement admises, lorsque les traitements $T I$ et $T 2$ sont appliqués, les plantes sont soit à l'état végétatif soit initiées. En ne tenant compte que de ces 2 étapes, $(A)$ il n'est pas possible d'expliquer la différence de réaction entre les plantes parasitées $T 1$ et T2. L'interprétation de ces résultats est par contre possible si on admet l'existence d'une étape préliminaire $(B)$.

\section{RÉFÉRENCES BIBLIOGRAPHIQUES}

Blondon F., 1968. Facteurs externes et déterminisme floral d'un clone de Dactylis glomerata $L$. Thèse Dr. ès Sci, CNRS A.O. 2522, $105 \mathrm{p}$.

Delecolle R., Gurnade J. C., 1980. Liaison entre le développement et la morphologie du blé tendre d'hiver. I. - Stades de développement de l'apex, apparition des feuilles et croissance de la tige. $A n n$. Amélior. Plantes, 30 (4), 479-498.

Duval E., Piton F., Regnault A., Sauvage M., 1981. Influence d'Ustilago hypodytes (Schlecht.) Fr. sur le tallage et la montaison de Bromus erectus Huds, Agronomie, 1 (3), 191-194.

Gay J. P., Menétrier M. A., 1978. Morphogenèse des organes reproducteurs chez le mais : échelle de notation des stades. - AGPMITCF, 8, avenue du Président Wilson, 75116 Paris, 38 p.
Jonard P., Koller J., Vincent A., 1952. Evolution de la tige et de l'épi chez la variété de blé Vilmorin 27 au cours de la période de reproduction. Ann. Amélior. Planies, 2, 31-54.

Malvoisin P., 1984. Organogenèse et croissance du maître-brin du blé tendre (Triticum aestivum) du semis à la floraison. II. - Contrôle des relations entre la croissance et la vascularisation de la tige et des feuilles. Essai de modélisation. Agronomie, 4 (7), 587-596.

Piton F., Duval E., Régnault A., Sauvage M., 1982. Effets du parasitisme d'Ustilago hypodytes (Schlecht.) Fr. sur le développement des tiges d'un clone de Bromus erectus Huds. Agronomie, 2 (4), 353-358.

Prat H., 1948. Histo-physiological gradients and plant organogenesis. Bot. Rev., XIV, 10, 603-643. 